\title{
USO DE LAS FICHAS DIDÁCTICAS EN V GRADO DE LA EDUCACIÓN PRIMARIA: VISIÓN DE LOS EDUCADORES EN SAN RAMÓN
}

\author{
Alicia Alfaro Valverde \\ Gilberto Chavarría Chavarría
}

Recibido 12-V-2003 • Aceptado 10-VI-2003

\begin{abstract}
Resumen: Este artículo es parte de un proceso de investigación desarrollado en el año 2001 en dos circuitos escolares de la Dirección Regional de Educación de San Ramón. Presenta los resultados obtenidos de los educadores de $\mathrm{V}$ grado en relación con el uso de las fichas didácticas en Educación Primaria.

La ficha didáctica es una técnica que se propone desde finales del siglo XIX, con el objetivo de individualizar la enseñanza y sigue manteniendo vigencia hasta el día de hoy. Por tal razón nos dimos a la tarea de investigar la concepción que manejan los educadores acerca de las fichas didácticas, las características, tipos, principios y valores que se pueden fortalecer, frecuencia con que se utilizan, razones de su uso, respuesta de los niños, ventajas, desventajas y sugerencias dadas por los educadores. Termina el artículo con algunas conclusiones en relación con la temática abordada.
\end{abstract}

Palabras clave: Educación, Didáctica, Ficha Didáctica, Enseñanza Individualizada, Técnica Didáctica.

\section{Introducción}

Este artículo es producto de un proceso de investigación desarrollado en el año 2001. La temática estudiada fue el uso de las fichas didácticas en $\mathrm{V}$ grado de la Educación Primaria. Dentro de esta investigación, conocer la opinión de los educadores acerca del uso y "manejo" de las fichas didácticas con niños de $\mathrm{V}$ grado de la escuela primaria, era uno de los propósitos fundamentales. De ahí que los objetivos que planteamos fueron los siguientes:

- Analizar críticamente la aplicación de las fichas didácticas para determinar las características que presentan, los tipos y la concepción de estas que "maneja" el educador.

- Determinar el nivel de aceptación por parte de los niños y la frecuencia con que se utilizan.

- Identificar los objetivos que se persiguen con el empleo de las fichas didácticas.

No cabe duda de que el acercarnos a conversar directamente con cada educador fue fundamental para observar reacciones y el interés que pueda despertar en ellos el tema y así conocer sus inquietudes y preocupaciones. Ese contacto con el docente fue 
muy productivo, pues nos permitió valorar desde la concepción de ficha didáctica que tiene el educador, hasta la aplicación de éstas y sus resultados. El análisis de estos procesos y de las condiciones en que se generan se convierten en el marco necesario para determinar de qué manera utilizan los educadores las fichas y cómo las han aplicado.

El siguiente apartado se iniciará con una breve caracterización de la población de educadores entrevistados. Luego se incorporan comentarios y tablas para establecer relaciones con cada una de las preguntas que le formulamos a los maestros. Se reconstruirán las experiencias de los docentes en torno al uso de las fichas, para indicar cuál es el sentir de éste acerca del "manejo" de ellas y las posibles sugerencias que puedan dar para el uso de esta técnica didáctica.

\section{Caracterización de la población participante}

\section{a. Centros educativos en los que trabajamos}

La población de educadores con la que trabajamos resultó muy heterogénea con respecto a los años de servicio (experiencia), procedencia según estudios superiores y en cuanto a las reacciones que pudimos observar cuando abordamos el tema en estudio.

Para desarrollar esta investigación trabajamos con 25 educadores que impartían en ese momento $\mathrm{V}$ grado en las diferentes escuelas del cantón de San Ramón. No trabajamos con educadores de escuelas unidocentes porque el sistema de fichas es uno de los empleados por ellos, debido a que tienen que atender más de un nivel al mismo tiempo e incluso se han elaborado materiales exclusivos para ellos.

La selección de los educadores de V grado fue intencional debido a la gran cantidad de contenidos que se imparten en este nivel, especialmente de Estudios Sociales y Ciencias, y a raíz de la experiencia que acumulamos en la supervisión de prácticas docentes. Así, trabajamos aplicando una entrevista semiestructurada a todos los maestros que impartían en ese momento el V grado en diez escuelas diferentes.

Los diez centros educativos seleccionados se encuentran ubicados de la siguiente forma. Escuelas públicas en el distrito central: Jorge Washington, José Joaquín Salas Pérez, Laboratorio, Alberto Manuel Brenes Mora. Las situadas en las cabeceras de distrito como: Monseñor Clodoveo Hidalgo (San Isidro), San Rafael y Federico Salas Carvajal (San Juan). Las otras escuelas que podríamos decir que se encuentran en áreas intermedias son las siguientes: Abrahan Paniagua Calvo (Calle Varela), Río Grande (La Guaria) y Nautilio Acosta Piepper (Esperanza de Piedades Norte).

\section{b. Centros educativos y cantidad de educadores}

Para llevar a cabo esta investigación seleccionamos un grupo de escuelas ubicadas geográficamente en el cantón de San Ramón de Alajuela. Dichos centros educativos están dentro de los circuitos 01 y 02 de la Dirección Regional de Enseñanza. Trabajamos con veinticinco educadores de quinto grado y los directores de las escuelas, para obtener la mayor cantidad de información posible. La distribución es la siguiente:

Tabla № 1

Distribución de los educadores por centro educativo

\begin{tabular}{lc}
\hline Centro educativo & Número de educadores \\
\hline Jorge Washington & 5 \\
José Joaquín Salas Pérez & 5 \\
Alberto Manuel Brenes Mora & 4 \\
Federico Salas Carvajal & 3 \\
Abrahan Paniagua Calvo & 2 \\
Nautilio Acosta Piepper & 2 \\
Clodoveo Hidalgo & 1 \\
Río Grande & 1 \\
San Rafael & 1 \\
Laboratorio & 1 \\
\hline Total & 25
\end{tabular}

Fuente: Entrevistas directas con educadores (agosto a octubre, 2001). 


\section{c. Años de servicio de los educadores}

Como puede observarse en la tabla № 2 , un $48 \%$ de los educadores cuentan con más de 10 años de servicio. Esto significa 10 o más años de experiencia, de compartir el trabajo con los niños, otros educadores y padres de familia. Si sumamos este porcentaje con un $32 \%$ que equivale a 8 educadores que en este momento han trabajado en educación más de 15 años, el resultado será de $80 \%$ de educadores que tienen entre diez y treinta años de impartir lecciones.

Tabla № 2

Años de servicio de los educadores entrevistados

\begin{tabular}{lcc}
\hline Intervalos & C.E.E. & $\%$ \\
\hline De 1 a 5 & 2 & 8 \\
De 5 a 10 & 3 & 12 \\
De 10 a 15 & 12 & 48 \\
De 15 a 20 & 3 & 12 \\
Más de 20 & 5 & 20 \\
\hline Total & 25 & 100 \\
\hline
\end{tabular}

Fuente: Entrevistas directas con educadores (agosto a octubre, 2001).

C.E.E.: Cantidad de educadores entrevistados.

\section{Las fichas didácticas y su uso}

De una manera muy resumida presentamos algunas de las definiciones en torno a lo que algunos autores entienden como ficha didáctica. Cabe aclarar que existen otras; pero en esta ocasión seleccionamos las de autores que están estrechamente relacionados con la enseñanza individualizada.

\begin{tabular}{ll}
\hline Di Rosa G. & "Es un medio, el mejor para adaptar la \\
& enseñanza a los escolares que la poseen \\
& y a las circunstancias concretas en las \\
& cuales se encuentran" (1974:31). \\
\hline F. Mory & "Es la preparación escrita del trabajo de \\
& clase concebida para determinado niño o \\
& niños y en sustitución de la lección expo- \\
& sitiva y rutinaria del maestro" (1964:5).
\end{tabular}

José Ml. "El sistema con fichas es eminentemente Valero psicológico porque responde a verdaderos intereses y necesidades del niño" (1975:71).

\begin{tabular}{ll}
\hline Adolfo & "La ficha de trabajo es el hilo conductor \\
Maello & que manda, dirige, motiva. No llega a \\
& ser un método; es un instrumento de \\
& trabajo que facilita la graduación del \\
& aprendizaje por unidades asequibles a \\
& los alumnos, respetando el nivel de ca- \\
& pacidad" (1968). \\
\hline Robert & "La ficha es un modo indirecto de mandar. \\
Dottrens & Es una táctica mediante la cual, el pro- \\
& pio alumno se ve responsabilizado a rea- \\
& lizar un trabajo señalado o insinuado \\
& por la ficha, sin presionarlo" (1973:40).
\end{tabular}

Ahora bien, Di Rosa (1974) deja claro que ofrecer una definición válida y completa de las fichas es imposible, lo que sí se puede hacer es dar características exteriores y decir que la ficha es una cartulina blanca o coloreada, ni muy gruesa ni muy fina.

Otro de los aspectos que le preocupa a dicho autor es dar una definición restrictiva de lo que es una ficha y que sea definida de manera absoluta; “...esto es, si se trata de algo fijo e inmutable o si, por el contrario, tiene el don de la adaptabilidad." (Di Rosa, 1974:31). Otro de los dones que le otorga a las fichas es la ductilidad, justificándola cuando dice que una misma clase de ficha asume características distintas en diferentes escuelas y que están sujetas a sufrir modificaciones.

Las fichas pueden ser contempladas como un recurso didáctico e instrumento de trabajo que permite el desarrollo de "una enseñanza individualizada que considera a cada niño como un ser muy especial, que necesita atención particular. Enfatiza de modo primordial la libertad, con sus limitaciones propias, que es necesaria para el desarrollo del individuo y para propiciar un ambiente de trabajo indispensable si verdaderamente se quiere practicar la enseñanza por acción”. (Barrantes, 1999:187).

Por otro lado, la ficha es una técnica de enseñanza muy útil, pues sirve de complemento a otras técnicas de enseñanza. 
Es una herramienta necesaria cuando se trabaja con alumnos que terminan más rápido que el resto de la clase y cuando existen estudiantes que requieren recuperación (Mujica, 1986:212-213).

Si consideramos las definiciones anteriores nos damos cuenta que todas responden a individualizar la enseñanza, viendo al ser humano de manera integral y como persona, sin dejar de lado las diferencias existentes entre los alumnos, pues tienen intereses y actitudes diversas.

Indudablemente las fichas de trabajo responden en gran medida a individualizar la enseñanza. Pero, ¿qué se pretendía con ello? Se buscaba que el alumno adquiriera los contenidos y formas de trabajo en forma independiente. (M.E.P. 1983:8). En algunos países europeos se planteaba la necesidad de estudiar e investigar lo relativo a la enseñanza colectiva donde era el maestro, casi siempre, el que tenía la última palabra, el que decidía qué contenidos, cuántos y cómo se debían impartir. De ahí que no fue una tarea fácil, es a partir de finales de la década de 1800 cuando el Dr. Decroly da inicio (1887), a lo que algunos han llamado "la renovación educativa en Bruselas". Esto marca cambios que tenían una sola dirección, individualizar la enseñanza. En este sentido surgen varias experiencias con el objetivo de satisfacer los intereses y necesidades de cada alumno y las características de la materia de aprendizaje. Entre estas experiencias Robert Dottrens propone la individualización por medio de fichas. $\mathrm{Al}$ respecto Dottrens manifiesta:

"Hemos mantenido, pues, la enseñanza colectiva, única que permite, a todo lo largo de la escolaridad, el intercambio y la formulación de ideas y que desarrolla el lenguaje y las facultades de expresión y simultáneamente, hemos introducido una enseñanza individualizada en la medida de lo posible". (Citado: MEP. 1983:10).

F. Mory, desde 1964, especificaba muy bien qué significa introducir la enseñanza, indicando que: “...para obtener del niño un esfuerzo fecundo, es necesario aprender a conocer sus intereses y explotarlos; luego, darle la ocasión de la actividad, de la creación, dejarle iniciativa y libertad. En fin, es necesario crear una corriente de confianza entre el maestro y el alumno; no de familiaridad, sino de comprensión". F. Mory insiste en que el alumno debe trabajar a su propio ritmo, anota que dentro de la organización práctica de la enseñanza individualizada pueden contemplarse las tareas y el trabajo con fichas, elemento esencial del trabajo individual. Esto va a permitir atender particularidades de los niños a los cuales hay que ayudar, especialmente para el caso de ciertos contenidos de difícil comprensión. Es así como nació el crear formas que permitieran individualizar los procesos. Con esta filosofía se inició lo que hoy día es parte del desarrollo de muchas lecciones a nivel escolar, donde se incorporan dentro de un mismo procedimiento a uno o más niños, muchas veces, sin considerar los niveles de dificultad y las características particulares de cada quien. Al homogenizar la enseñanza se corren riesgos muy grandes, en este caso con el uso de las fichas, pues con frecuencia se observa el uso de ellas sin tener en cuenta que estas “...deben estar adaptadas a la clase y redactadas por el maestro para sus alumnos. Debe mantener el tono familiar de la conversación y dirigirse directamente al alumno". (F. Mory, 1964: 6).

Dottrens, al respecto indica:

\begin{abstract}
"Creemos en el valor de las fichas, en la medida en que corresponden a las necesidades sentidas en la enseñanza (fichas de recuperación), a intereses manifestados por los niños (fichas de ejercicios y de desarrollo), a las condiciones y circunstancias en las cuales se da la enseñanza; sean cuales fueren, las fichas deben actualizarse lo más que sea posible." (Dottrens, 1973:28).
\end{abstract}

Insiste Dottrens en la no estandarización de las fichas, por el contrario rechaza la idea de que existan fichas valederas para todos y en todas partes. 
Ya en las tres primeras décadas del siglo pasado se comienza a pensar más fuertemente en las diferencias y el poder tener en cuenta las capacidades de los alumnos.

En los Estados Unidos de América, por ejemplo, se desarrollaron experiencias donde se pretendía fortalecer la libertad del niño, es el caso del Plan Dalton, promovido por Helen Parkhurst; también el Sistema Winnelka que promovía el tener muy en cuenta los intereses de los niños que se podían conocer, utilizando para ello diagnósticos.

Las ideas del Plan Dalton y del Sistema Winnelka son consideradas por Robert Dottrens para plantear la individualización de la enseñanza en la escuela primaria. Así, este pedagogo francés propone diferentes tipos de fichas, entre las que están las de instrucción, cuya finalidad es que los alumnos obtengan información, conozcan conceptos nuevos, principios y valores para $\mathrm{su}$ vida futura. En segundo lugar, las fichas de ampliación que pretenden profundizar los conocimientos sobre determinado tema. Luego aparecen las de ejercicios donde se busca que el alumno realice trabajos como tareas, cuestionarios, ubicación en los mapas, resolución de problemas, etc. Y por último, las de recuperación que buscan superar los problemas detectados en el proceso de enseñanza-aprendizaje que experimenta el alumno.

\section{El educador de $\mathrm{V}$ grado de Educación Primaria y las fichas didácticas}

\section{a. Concepción de ficha que "manejan" los educadores}

En algunos textos como Educación Personalizada de Valero (1975), las guías y fichas responden al mismo propósito. Este autor define las guías o fichas de la siguiente forma: "En su totalidad forman un sistema de enseñanza programada, el cual debe ser consultado para su uso adecuado y correcto." (Valero, 1975: 70).

A este mismo autor le interesa dejar claro que las fichas no son un método, sino por el contrario un procedimiento que busca personalizar el aprendizaje.

Ahora bien, qué definiciones mencionan los maestros acerca de lo que es una ficha.

Tabla № 3

Conceptualización del término ficha, según los educadores

Conceptos o definiciones

C.E.E

Son una forma de trabajo que tiene estructura y objetivos, aunque no estén explícitos. Además, deben ser planeadas, organizadas y no pueden ser improvisadas. Dicho trabajo se puede realizar en las diferentes áreas para retroalimentar y evaluar los contenidos.

Son recursos complementarios y valiosos de los cuales se vale el educador para que el alumno fabrique el conocimiento. Como recurso las fichas también son documentos que le sirven al docente para reforzar, practicar y verificar contenidos. Además, son una ayuda didáctica para el trabajo con los niños.

Son materiales de apoyo que sirven para motivar, reforzar, desarrollar el tema y que cuentan con muchas utilidades más, como evaluar contenidos.

Son una apoyo, un refuerzo y una motivación. 6

Son instrumentos complementarios a la hora de desarrollar un tema. Sirven para ampliar y desarrollar una lección. Permiten alcanzar objetivos, para ampliar y concluir un tema.

Son guías de trabajo para combinar el trabajo con la pizarra.

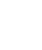

Son una técnica de trabajo, tienen pasos por desarrollar y su metodología, que muchos educadores no dominan.

Fuente: Entrevistas directas con educadores (agosto a octubre, 2001).

C.E.E.: cantidad de educadores entrevistados. 
Como puede observarse en la tabla anterior los educadores concuerdan al afirmar que las fichas son un recurso, instrumento, material, guía de trabajo o un apoyo en el proceso enseñanza-aprendizaje. Es evidente que las definiciones de ficha que dan los maestros en alguna medida responden también a lo señalado por Dottrens (1959), cuando se refiere a la ficha como un procedimiento útil para la enseñanza.

Veamos en el esquema siguiente cuáles son las palabras con las que los educadores asocian las fichas.

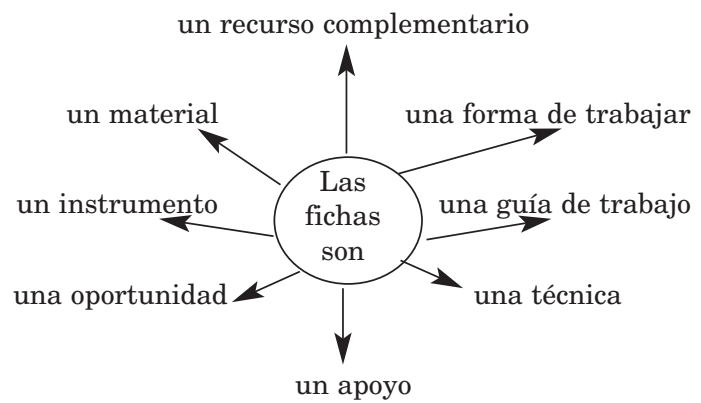

Lo anterior, más las cifras de la tabla № 3 nos demuestran que los educadores conceptualizan las fichas con diversidad de nombres que dentro del contexto educativo son completamente válidos, puesto que existen muchas definiciones acerca de lo que es una ficha. Como bien lo apunta Di Rosa (1974), es imposible ofrecer una definición completa y válida de lo que es una ficha.

\section{b. Características de las fichas didácticas}

Cuando leemos a Valero y a Dottrens, quienes se refieren a las características que debe tener una ficha didáctica para que se le reconozca como tal, nos damos cuenta de que los educadores no precisan exactamente lo que los autores hablan, pero sí con mucho acierto indican una serie de características que sí corresponden con la teoría acerca de las fichas.
Tabla № 4

Características de las fichas, según los educadores

Características de las fichas

C.E.E

Deben contener algunos datos generales co-

23

mo: objetivos, instrucciones, tema, área, etc.

Deben ser de un tamaño pequeño.

8

Que tengan claridad en cuanto a los contenidos y que se siga el orden lógico de la materia.

Deben ir paso a paso para que el niño logre lo que se pretende.

6

Conviene que las fichas sean atractivas para animar al niño.

6

Deben ser concretas y no estar recargadas.

Con un tamaño regular. 3

Que incorporen dibujos. 3

Que se elaboren con letra grande. $\quad 2$

Que estén dentro del contexto en el que se $\quad 2$ desenvuelve el niño.

Otras *

5

(*) Corresponde a otras características que fueron mencionadas cada una por un docente, entre ellas: las fichas deben ser elaboradas con letra legible, tener unidad de pensamiento, encabezado, que se presenten solo por un lado y con un material consistente.

Fuente: Entrevistas directas con educadores (agosto a octubre, 2001).

C.E.E.: cantidad de educadores entrevistados.

Las características que indican los educadores de una u otra forma ya están dadas por quienes han escrito acerca de las fichas didácticas. Por ejemplo, Valero y Dottrens se refieren al tamaño y estructura de las mismas indicando que es preferible al elaborarlas utilizar cartulina o sea un material consistente y con un tamaño pequeño (quince por veinte centímetros); que se presenten bien atractivas, con dibujos, gráficos; que contengan un vocabulario 
al alcance de los niños y “... que se adapten a las necesidades propias de cada grupo, al medio en que se aplican y a los recursos existentes." (M.E.P. 1983:54).

De acuerdo con lo anterior, podemos afirmar que la mayoría de los educadores entrevistados considera que las fichas son un trabajo que requiere planearse y además tener muy en cuenta una serie de aspectos como objetivos, instrucciones, tema, área, entre otros.

\section{c. Tipos de fichas}

De acuerdo con la información suministrada por los educadores (tabla №5), los tipos que ellos mencionan concuerdan en gran medida con las clasificaciones que existen en torno a las fichas. Sin embargo, en algunos casos, como bien se evidencia en la tabla sugerida, la mayoría de los educadores (20) expresa como un tipo de ficha las elaboradas por el Programa de Mejoramiento de la Calidad de la Educación (PROMECE), las cuales evidentemente son utilizadas por ellos.

Cabe indicar que después de hacer una revisión de las fichas de PROMECE, se puede afirmar que para cada nivel existe un buen número de ellas y que fueron elaboradas siguiendo alguna clasificación, por esa razón hay diferentes tipos en cada juego de fichas que utiliza el maestro. De acuerdo con esto, PROMECE no responde exactamente a un tipo de ficha, sino al nombre de un programa que, entre otras actividades, publica este material.

Ahora bien, la tabla № 5 también nos revela que los nombres dados por los educadores corresponden con algún tipo de ficha, solo que el maestro la llama de ese modo, básicamente por el hecho de que se relaciona con lo que le asignará al niño o al contenido de la ficha misma. Por ello consideramos todos los nombres dados por los educadores, aún cuando, por ejemplo, en ejercicios podríamos ubicar también las fichas de actividades (observar, narrar,
Tabla № 5

Tipos de fichas, según los educadores

Tipos de fichas C.E.E.

C.E.E

\begin{tabular}{lc}
\hline Promece & 20 \\
\hline Refuerzo & 18 \\
\hline Motivación & 12 \\
\hline Ejercicios & 10 \\
\hline Guía & 10 \\
\hline Investigación & 10 \\
\hline Contenidos & 9 \\
\hline Información & 9 \\
\hline Documentación & 9 \\
\hline Extraclase & 8 \\
\hline Evaluación & 7 \\
\hline Prácticas & 7 \\
\hline Resumen & 6 \\
\hline Repaso & 5 \\
\hline Otras * & 6 \\
\hline
\end{tabular}

(*) Corresponde a otros tipos que fueron mencionados por los educadores. Cada una por un maestro, entre ellos actividades, conocimiento, recreación, tarea, juegos y cuestionarios.

Fuente: Entrevistas directas con educadores (agosto a octubre, 2001).

C.E.E.: cantidad de educadores entrevistados.

buscar, calcular, describir, dibujar, etc.), las de prácticas, juegos, tareas, cuestionarios, recreativas, entre otras. En las de investigación se pueden incluir aquellas que llevan a la observación, experimentación, documentación y desarrollo. En las de autoinstrucción se podrían anotar las de razonamiento y reflexión.

Es muy importante tener en cuenta que a raíz de la ductilidad de las fichas, en ocasiones existen semejanzas entre los diferentes tipos; sin embargo, ocurre como en el caso de las fichas de investigación y ejercitación, una relación por secuencia, donde la investigación se presenta antes de la lección y ayuda a prepararla, mientras que las fichas de ejercitación la siguen y concluyen.

Revisando cuadernos de los niños (diez en total), nos damos cuenta de la cantidad, tipo de ficha que aparecen pegadas y en la disciplina que se trabajaron. 
La tabla № 6 indica cómo lo que está en los cuadernos de los niños coincide con las entrevistas que hicimos a los maestros, pues ellos mencionaron que hacen uso de diversas técnicas, utilizando entre ellas las fichas, pero escasamente. Esto, más lo que dijeron los niños y la revisión de cuadernos, nos conduce a afirmar que el maestro elabora muy pocas fichas didácticas.

En cuanto a las fotocopias que no siguen ningún formato de ficha didáctica, sí se utilizan más por los educadores. Esto se observa en los cuadernos de los niños. En la tabla № 6 se puede apreciar que es en Ciencias y Estudios Sociales donde más se emplean las fotocopias. En Ciencias, principalmente, para estudiar lo relativo a los sistemas (respiratorio, digestivo, reproductor, etc.) y en Estudios Sociales para abarcar lo relativo a la historia de Costa Rica.

Cabe indicar que los niños a la luz de lo que aparece en las fotocopias (lecturas, cuentos, mapas, figuras humanas, esquemas, crucigramas, palabragramas, ejercicios de completar, de asociación, cuestionarios, resúmenes, ejercicios matemáticos como problemas, completar cuadros, figuras o dibujos, resolver operaciones básicas, etc.) debían hacer el trabajo en ese momento o extra clase. De acuerdo con lo visto en los cuadernos y como bien se indica en la tabla № 15, el educador combina mucho el dibujo, la imagen y el texto. También se utilizan bastante los cuestionarios que el niño responde con la ayuda de textos incluidos en fotocopias o el libro de texto, básicamente los libros de Santillana, Farben y Norma, algunos recortes de periódico (El Heraldo), escasamente el libro Serie: Hacia el siglo XXI y las fichas de PROMECE que, como bien se puede apreciar en la tabla № 6 , son utilizadas por los educadores, no abundantemente; pero sí en ocasiones, principalmente para el desarrollo de algún tema o para reforzar. Sin embargo, los cuadernos revisados nos confirman que evidentemente no hay abuso al usarlas.

\section{d. Principios y valores que se for- talecen con el uso de las fichas}

Según los educadores, son varios los principios y valores que se pueden desarrollar con el uso de las fichas, entre ellos la cooperación, compañerismo, responsabilidad, libertad, creatividad, autonomía, orden,

Tabla № 6

Cuadernos de los niños de $\mathrm{V}$ grado que fueron considerados

\begin{tabular}{llcccc}
\hline C.C & \multicolumn{1}{c}{ Área } & CPEP & Fotocopias & $\begin{array}{c}\text { Fichas } \\
\text { PROMECE }\end{array}$ & Fichas elaboradas por el maestro \\
\hline 1 & Estudios Sociales & 71 & 26 & 11 & 5 (de documentación, refuerzo, \\
& & & & - & - \\
2 & Estudios Sociales & 10 & 10 & 8 & - \\
3 & Ciencias & 51 & 31 & - & 4 (de refuerzo, guía y secuencias) \\
4 & Ciencias & 40 & 5 & 5 & 2 (de actividades) \\
5 & Ciencias & 81 & 45 & - & 3 (de repaso y documentación) \\
6 & Matemática & 26 & 9 & 8 & 3 (de refuerzo y actividades) \\
7 & Estudios Sociales & 45 & 19 & 1 & 2 (de actividades) \\
8 & Español & 112 & 17 & 1 & -15 \\
9
\end{tabular}

Fuente: cuadernos de niños de diferentes centros educativos, facilitados por ellos y con el debido permiso de los padres de familia, 2001.

(*) En matemáticas también revisamos el cuaderno de tareas y con ejercicios muy parecidos a los que los niños resuelven en clase.

Notas: C.C = cantidad de cuadernos.

CPEP = cantidad de páginas escritas por los niños. 
aseo y que los niños puedan razonar con la puesta en práctica de esta técnica.

De tal forma que un $68,2 \%$ de los maestros encuentra que sí se desarrollan principios y valores como los ya citados, mientras que un $18,2 \%$ indica que a veces se pueden lograr ciertos principios y que esto depende del tipo de ficha y el 13,6\% restante opina que realmente con las fichas lo que se fomenta es la vagancia de los niños.

De acuerdo con el personal docente de la Escuela Patriarca San José (1979), la ficha:

"Favorece la formación de hábitos de destrezas, de desenvolvimiento, de concentración, de lucha ante la dificultad, el espíritu de observación, la ocasión de manifestar su originalidad y hace al alumno más responsable." (E.P.S.J. 1979:17).

Lo anterior coincide en alguna medida con lo que exponen los educadores cuando nos indicaron que dentro de los principios y valores educativos que las fichas fomentan están los siguientes:

\section{Tabla № 7}

Principios y valores que se pueden desarrollar con el uso de las fichas, según los educadores

\begin{tabular}{lc}
\hline \multicolumn{1}{c}{ Principios y valores } & C.E.E \\
\hline Cooperación y compañerismo & 12 \\
Responsabilidad & 8 \\
Orden y aseo & 7 \\
Libertad & 5 \\
Autonomía & 5 \\
Creatividad & 5 \\
Razonamiento & 5 \\
Socialización & 3 \\
Honestidad & 1 \\
\hline
\end{tabular}

Fuente: Entrevistas directas con educadores (agosto a octubre, 2001).

C.E.E.: cantidad de educadores entrevistados.

\section{e. Frecuencia con la que los educadores utilizan las fichas}

Es interesante indicar que un $88 \%$ de los educadores opina que la frecuencia con la que se pueden utilizar las fichas didácticas varía e incluso hay días que no se usan, pues se trabaja con otras técnicas como la expositiva, interrogativa, experimentos, demostraciones, juegos, dinámicas o con la ayuda del libro de texto y de fotocopias, que no tienen las características de una ficha. También algunos educadores insistieron en que la frecuencia con que se utilice una u otra técnica de trabajo depende mucho del educador y que por ello varía desde no utilizarla todos los días, hasta tres veces al día o solo para asignar un trabajo extraclase, donde los niños hagan investigación. (Ver tabla № 8).

De acuerdo con los datos de la tabla № 8, 18 maestros mencionaron que depende del tema y de la asignatura. Estudios Sociales y Ciencias son las áreas donde más se emplean las fichas, no quedando muy rezagadas Español y Matemáticas.

Con la revisión de los cuadernos se comprueba aún más que en estas disciplinas es donde más se utilizan las fichas. (Ver tabla № 6).

Tabla № 8

Frecuencia con la que se utilizan las fichas, según los educadores

\begin{tabular}{lc}
\hline \multicolumn{1}{c}{ Frecuencia } & C.E.E \\
\hline Hay días que no se usan. & 24 \\
Depende del tema y de la asignatura. & 18 \\
Depende del ciclo, en I grado se usan más, & \\
por lo de apresto, en realidad se usan más & \\
en I ciclo. & 15 \\
Una ficha por día, máximo dos. & 10 \\
No todos los días. & 9 \\
Depende del docente. & 6 \\
Moderadamente, solo por un período. & 6 \\
No muchas, solo para reforzar y motivar. & 5 \\
Hasta tres fichas al día como refuerzo. & 2 \\
Otras * & 7 \\
\hline
\end{tabular}

(*) Corresponden a una razón por maestro, entre las cuales están: máximo 3 ó 4 a la semana, como trabajo extraclase para que los niños investiguen, lo que se usa muy frecuentemente es la fotocopia, se usan hoy más que ayer, las usa por la tarde, se utilizan más en I grado y las usa de manera equilibrada.

Fuente: Entrevistas directas con educadores (agosto a octubre, 2001).

C.E.E.: cantidad de educadores entrevistados. 


\section{f. ¿Toda hoja fotocopiada es una ficha?}

Cuando le preguntamos a los educadores si toda hoja fotocopiada es una ficha las respuestas que obtuvimos fueron las siguientes: 22 (88\%) indicaron que no, $2(8 \%)$ no respondieron y 1 (4\%) dijo que sí. Estos datos se relacionan con las características de las fichas mencionadas por los educadores. Al respecto (23) manifestaron que un documento para llamarse ficha requiere de ciertos datos como objetivos, instrucciones, tema, área entre otros y que, por lo tanto, no puede ser un documento improvisado, puesto que tiene que ser claro, sin ambigüedad, atractivo para los niños y no tener un tamaño muy grande. Una de las educadoras nos dijo: "un documento para que se llame ficha, hay que elaborarlo teniendo en cuenta el grado de dificultad del niño. (Cárdenas, octubre, 2001).

Las razones expuestas por los educadores coinciden con las características de las fichas indicadas por diferentes autores en páginas anteriores, aún cuando el 100\% de los maestros mencionaron no haber recibido nunca ningún tipo de charla, taller o asesoramiento en torno a la confección, aplicación y evaluación de las fichas didácticas y manifestaron que muchas veces se desconocen las características y condiciones que requiere un documento para conceptualizarse como ficha.

\section{g. Uso frecuente de las fotocopias ${ }^{1}$ : razones que lo justitican}

Al preguntar a los educadores por qué es frecuente el uso de fotocopias con los niños, estos indicaron una serie de razones expuestas en la tabla № 9 .

Los educadores indican aspectos muy importantes como la falta de tiempo, la abundancia de fotocopiadoras, la comodidad y facilidad y el mismo hecho de que elaborar las fichas lleva mucho trabajo.
Tabla № 9

Razones que justifican el uso frecuente de las fotocopias, según los educadores

Razones

C.E.E

La cantidad de contenidos. Los programas del M.E.P. están recargados y aparecen muchos contenidos repetidos para los exámenes de sexto grado.

Por falta de tiempo y porque se atrasó la materia.

15

Por el hecho de que hay fotocopiadoras por todos lados.

\begin{tabular}{ll}
\hline Por comodidad y facilidad. & 7 \\
\hline Porque hacer las fichas lleva mucho trabajo. & 5 \\
\hline $\begin{array}{l}\text { Debido a que las fotocopias sirven para salir } \\
\text { del paso y para adelantar materia. }\end{array}$ & 5 \\
\hline $\begin{array}{l}\text { A raíz de la gran cantidad de trabajo que tie- } \\
\text { ne el maestro y al hecho de que en lugar de } \\
\text { trabajar por objetivos lo hace por contenidos. }\end{array}$ & 5 \\
\hline Debido a la actitud del maestro. & 2 \\
\hline $\begin{array}{l}\text { Por pereza. } \\
\text { Por quedar bien con los padres de familia. }\end{array}$ & 1 \\
\hline $\begin{array}{l}\text { Debido a que algunos maestros quieren sen- } \\
\text { tirse más modernos. }\end{array}$ & 1 \\
\hline
\end{tabular}

Fuente: Entrevistas directas con educadores (agosto a octubre, 2001).

C.E.E. cantidad de educadores entrevistados.

Por otro lado, aparecen justificaciones que corresponden con la actitud del educador, la responsabilidad y el interés que demuestra al trabajar con niños.

Es interesante tener en cuenta que el examen de sexto grado, incide mucho para que se utilicen gran cantidad de fotocopias. Según ellos, deben correr para abarcar toda la materia.

\section{h. Respuesta de los niños ante el uso de las fichas didácticas}

Según los educadores, a los niños les gusta trabajar con fichas (ver tabla № 10), principalmente cuando estas traen dibujos para colorear o los ejercicios que contienen algún experimento. Las fichas que son solo resumen o cuestionarios no son tan del agrado de los niños, ellos prefieren que el 
maestro les explique y que las lecciones sean más creativas y dinámica.

\section{Tabla № 10}

Respuesta de los niños ante el uso de las fichas, según los educadores

\begin{tabular}{lc}
\hline Apreciaciones & C.E.E \\
\hline A los niños les gustan & 21
\end{tabular}

A algunos no les gustan, reclaman cuando son muchas.

Fuente: Entrevistas directas con educadores (agosto a octubre, 2001).

C.E.E.: cantidad de educadores entrevistados.

\section{i. Reacción de los padres de familia en torno al uso de las fichas}

La reacción de los padres de familia con respecto al uso de las fichas didácticas con los niños, en términos generales es de rechazo, pues al tener en cuenta las cifras de la tabla № 11, podemos darnos cuenta de que a los padres de familia, (16 de ellos) no les gusta que se utilicen tantas fichas. En primer lugar, por el gasto en dinero y, en segundo lugar, porque se desperdicia el cuaderno que "solo se usa para pegar y pegar hojas". A su vez los padres se quejan de lo desagradable que se ve el cuaderno y de la cantidad de fichas de contenido que recargan mucho al niño para estudiar para los exámenes. Algunas de las madres de familia entrevistadas manifestaron lo siguiente:

- $\quad$ "Se usan muchas fichas, que más bien son fotocopias con mucho contenido. Esto le cuesta mucho al niño para estudiar, porque tiene que estudiar a veces materia que el maestro no ha explicado, solo se la dio." (Campos, octubre, 2001).

- $\quad$ "Sí se usan mucho las fotocopias, a veces hasta tres páginas por día y qué pasa con los niños lentos y de adecuación curricular." (Valerio, agosto, 2001).
- “Sí se usa mucho, se centra en solo ese tipo de trabajo. Mi hijo se atiene a eso y cada vez se vuelve más perezoso." (Herrera, agosto, 2001).

- "Sí se usa mucho, con frecuencia a mí me preocupa el que solo peguen hojas en el cuaderno." (Jiménez, agosto, 2001).

- "Con tantas fotocopias el niño no domina para el examen. Yo conozco niños que no van a clase y sacan las copias y las pegan en el cuaderno, o chiquitos que no copian en clase y solo van y pegan." (Palma, agosto, 2001).

\section{Tabla № 11}

Reacción de los padres de familia con respecto al uso de las fichas didácticas y fotocopias, según los educadores

Reacciones de los padres de familia C.E.E.

No les gusta mucho debido al gasto económico.

No les gusta mucho porque es un desperdicio, pues no se usa el cuaderno y además se ve feo.

Se quejan porque hay cuadernos que son solo fotocopias con contenidos y nada de prácticas.

Hace falta la explicación del maestro y en realidad les gustan los cuadernos donde el niño trabaja con lecturas y dibujos.

Depende el tipo de papá, de la educación y posibilidades económicas que tengan.

A los papás no les gustan las cosas fáciles, ellos prefieren que no se usen las fichas y hablan de los maestros que las usan.

No tiene problemas con los papás, les gustan mucho y envían el dinero para sacar las fotocopias. 2

A los papás las fichas les gustan a medias. 2

Fuente: Entrevistas directas con educadores (agosto a octubre, 2001).

C.E.E. : cantidad de educadores entrevistados. 


\section{j. Ventajas del uso de las fichas di- dácticas}

Dentro de las ventajas que tiene el trabajo con fichas, según los educadores, una de las más relevantes es el hecho de que con el empleo de esta técnica se economiza tiempo. Por otro lado, con el empleo de las fichas didácticas se refuerzan contenidos, se facilita el trabajo y se enriquece el aprendizaje con la aplicación de diferentes ejercicios que se incluyan en las mismas. Además, abren la posibilidad de enriquecer los contenidos para de esta manera avanzar más rápido y poder atender las diferencias individuales. Veamos la siguiente tabla que contiene las ventajas mencionadas por los educadores.

\section{Tabla № 12}

Ventajas del uso de las fichas didácticas, según los educadores

\begin{tabular}{lc}
\hline \multicolumn{1}{c}{ Ventajas } & C.E.E. \\
\hline $\begin{array}{l}\text { Con el empleo de las fichas se economiza tiempo. } \\
\text { Por medio de las fichas se puede reforzar el } \\
\text { conocimiento. }\end{array}$ & 13 \\
\hline $\begin{array}{l}\text { Las fichas facilitan el trabajo, pues permiten ha- } \\
\text { cer esquemas, resúmenes y ampliar contenidos. }\end{array}$ & 8 \\
\hline $\begin{array}{l}\text { Con el uso de las fichas se pueden enriquecer } \\
\text { los contenidos. }\end{array}$ & 7 \\
\hline $\begin{array}{l}\text { Con la ficha se puede salir de la rutina y ha- } \\
\text { cer menos tedioso el aprendizaje. El niño rápi- } \\
\text { do puede estar ocupado y desarrollando un } \\
\text { trabajo más activo. }\end{array}$ & 5 \\
\hline $\begin{array}{l}\text { Bien montada la ficha, el niño puede lograr ma- } \\
\text { yor hábito de la lectura y el niño la disfruta. }\end{array}$ & 4 \\
\hline $\begin{array}{l}\text { Las fichas le permiten al educador hacer repa- } \\
\text { sos para los exámenes. }\end{array}$ & 2 \\
\hline $\begin{array}{l}\text { Los niños pueden expresar más y dependiendo } \\
\text { de la ficha puede construir el conocimiento. }\end{array}$ & 2 \\
\hline \begin{tabular}{l} 
Otras * \\
\hline
\end{tabular}
\end{tabular}

(*) Corresponde a: las fichas sirven para ponerse al día, permiten hacer evaluaciones, ayudan en el control de la disciplina, son muy útiles para asignar trabajos extraclase, ayudan a los niños lentos para escribir y por medio de ellas los niños pueden compartir. Cada una de estas ventajas fue mencionada por un educador.

Fuente: Entrevistas directas con educadores (agosto a octubre, 2001).

C.E.E.: cantidad de educadores entrevistados.

\section{k. Desventajas del uso de las fichas}

Como bien se aprecia en la tabla № 13, del trabajo con fichas, según los educadores, también existen desventajas. Resulta interesante tener en cuenta que la mayoría (19 educadores) encuentra que con el uso de las fichas el alumno no quiere hacer casi nada, se vuelve "vago" y, lo que es más grave, no desea ni leer ni escribir. Como consecuencia directa de esto se da una redacción y ortografía de muy baja calidad. Por otra parte, el niño busca hacer las cosas más sencillas y donde tenga que hacer el menor esfuerzo. Por ejemplo colorear, enmarcar, recortar y pegar en los cuadernos entre otras actividades.

Lo anterior se ve reforzado por el uso promedio o indiscriminado de fichas o fotocopias ${ }^{2}$, con lo cual muchas veces se cae en el "fichismo" que conduce a un gasto económico elevado para los padres de familia.

Con el uso abundante de las fichas se fomenta la copia que no genera producción por parte de los alumnos. El aprendizaje se vuelve pasivo y muy tedioso. 
Tabla № 13

Desventajas que tiene el uso de las fichas, según los educadores

$$
\text { Desventajas }
$$

C.E.E.

Los niños escriben poco al día, se hacen vagos, perezosos y dependientes de las fichas. Pierden escritura, por lo tanto se dan muchos problemas de ortografía y caligrafía.

Se abusa de las fichas, se hacen muchas, se cae en el "fichismo", tiene que ver la moda.

\begin{tabular}{ll}
\hline Los niños no quieren leer. & 12 \\
\hline $\begin{array}{l}\text { Los niños solo desean colorear, enmarcar } \\
\text { y pegar las fichas en el cuaderno. }\end{array}$ & 9 \\
\hline El gasto económico que resulta es alto. & 8 \\
\hline Fichas mal confeccionadas. & 7 \\
\hline $\begin{array}{l}\text { La ficha ha decaído por el uso de las foto- } \\
\text { copias. }\end{array}$ & 6 \\
\hline
\end{tabular}

Se fomenta la copia, pues todo se les da hecho, el aprendizaje se vuelve pasivo y muy descriptivo.

Los educadores no tienen claro qué es una ficha didáctica.

Hay que dedicar mucho tiempo para hacerlas.

Si se trabaja en grupos no todos participan. 3

Fuente: Entrevistas directas con educadores (agosto a octubre, 2001).

C.E.E.: cantidad de educadores entrevistados.

\section{Sugerencias dadas por los educadores en torno al uso de las fichas}

No cabe duda de que las sugerencias dadas por los educadores en cuanto al uso de las fichas didácticas son de gran valor no solo por la experiencia al trabajar con niños, sino también porque conocen la necesidad de presentarles a los educandos trabajos diversos, creativos y atractivos.
Tabla № 14

Sugerencias para el uso de las fichas, según los educadores

Sugerencias

C.E.E.

Deben ser elaboradas por los educadores y no simples fotocopias. Muchas cosas que vienen en los libros no son necesarias.

No abusar de ellas. 17

Las fichas deben ser bien planeadas y corresponder a ciertos objetivos, no una hoja con simple información.

Es conveniente que las fichas sean bien creativas, prácticas, con vocabulario sencillo y que promuevan la investigación.

Es necesario que las fichas correspondan con la realidad del niño y con el contexto en el cual este se desenvuelve.

Utilizar las fichas didácticas con los niños más rápidos.

5

Que las fichas no sustituyan al libro de texto.

5

Se le debe dar al alumno para que trabaje y que luego la devuelva.

No acostumbrar a los niños a trabajar solo con fichas.

5

No es conveniente que la ficha sirva solo para sacar de apuros.

Que se utilicen para trabajos extraclase. 4

No deben ser muy extensas, especialmente las de actividades.

El educador debe aclarar dudas antes de aplicarla y explicar muy bien el trabajo por resolver.

3

Deben ser utilizadas para trabajo individual.

Crear un banco de fichas didácticas en las escuelas.

Que se utilice solo en casos de emergencia.

Combinar el uso de las fichas con otras técnicas.

\section{1}

Fuente: Entrevistas directas con educadores (agosto a octubre, 2001).

C.E.E.: cantidad de educadores entrevistados. 
La tabla № 14 refleja que 18 de los educadores concuerdan al afirmar que las fichas deben "montarse", porque muchas de las cosas que vienen en los libros no son necesarias o son simplemente para ampliar y no conviene darle todo al niño.

También 17 de los educadores concuerdan en que no se debe abusar del uso de las fichas didácticas. Por el contrario se recomienda emplearlas con medida, en caso de emergencias y con los niños más rápidos, principio fundamental dado por los autores que se refieren al trabajo con fichas didácticas, entre ellos Dottrens y Valero.

$\mathrm{Si}$ revisamos las sugerencias dadas por los educadores, queda claro que existen una serie de cuidados que se deben tener al aplicar esta técnica. Desde la necesidad de elaborar las fichas teniendo en cuenta una serie de detalles como: los objetivos, el niño y el contexto en el cual se desenvuelve, ciertas condiciones que pueden tener las fichas (tamaño, vocabulario, letra, etc.), la conveniencia de que las fichas sean utilizadas de manera individual y que no se conviertan en un instrumento que sirva solamente para sacar de apuros.

Se extrae de esta misma tabla que los educadores conocen aspectos importantes del uso y manejo de las fichas didácticas y que es necesario que esta técnica se alterne con otras actividades para no cansar al niño.

Según la revisión de cuadernos, dentro de las actividades que llevan a cabo los educadores en el aula, están las siguientes:

- Que los niños desarrollaron directamente en las páginas de los cuadernos: lecturas con algunos ejercicios de comprensión (responder preguntas, hacer dibujos, redactar oraciones, crear una historia, subrayar términos, etc.), completar un cuadro, resolver crucigramas y palabragramas, dar nombres a diversas figuras y a las partes de las mismas, asociar y parear, colorear mapas, dibujar pirámides, resolver estudios dirigidos, elaborar mapas conceptuales, responder una serie de preguntas en forma de cuestionario, buscar palabras en el diccionario, buscar información en el libro de texto para pasarla al cuaderno o responder algunas preguntas, comentar frases, ir respondiendo guías de trabajo, construir oraciones, llevar a cabo identificaciones en diferentes figuras, desarrollar experimentos, prácticas con problemas, resolver ejercicios matemáticos, elaborar gráficos de barras y pastel, construir o dibujar figuras geométricas, resolver problemas haciendo uso de las operaciones básicas, llevar a cabo prácticas con palabras claves para estudiar los temas de Español (identificar letras dentro de oraciones, ubicar correctamente las palabras para dar sentido a la oración, etc.), entre otras.

- Que se desarrollaron en fotocopias y que fueron resueltas, coloreadas, enmarcadas y pegadas en el cuaderno: identificar y dar nombres a las imágenes que aparecen en las copias, por ejemplo en Ciencias lo relacionado con los reinos. Los niños también dibujan, asocian, completan frases, resumen, investigan o buscan en el diccionario o libro de texto, pegan recortes de periódico, resuelven crucigramas, palabragramas, marcan con (X) lo correcto, ubican en el mapa, colorean mapas, completan cuadros para hacer clasificaciones, resuelven cuestionarios en subgrupos, lecturas para contestar ejercicios de comprensión, lecturas para reflexión, dan títulos a imágenes, trabajos dirigidos, resolución de problemas matemáticos, cálculo de cantidades, ejercicios de respuesta breve, resolver ejercicios matemáticos como problemas, divisiones, multiplicaciones, completar figuras y cuadros con los resultados obtenidos, construyen narraciones, crean historias, leen poesía y desarrollan comentarios por escrito, leen versos, subrayan con color 
las palabras claves, con la ayuda de recortes de periódico crean oraciones y ubican palabras claves, lectura para obtener ideas principales y secundarias y para identificar el tipo de lectura que es, extraer palabras de los textos y clasificarlas, utilizando secuencias con dibujos para ir creando un cuento, entre otras cosas.

- Que se desarrollaron por medio de las fichas elaboradas por los educadores: hoja de cotejo, resúmenes y dibujos, cuestionarios, guías con la ayuda del libro de texto o una separata, observar secuencias y anotar al lado su significado, contestar preguntas en el cuaderno, resolución de problemas, resolución de crucigramas y palabragramas, completar oraciones o frases después de una lectura, reflexionar en torno a una lectura, subrayar palabras claves, resolver problemas matemáticos, ejercicios con las operaciones fundamentales, con el sistema de numeración, completar cuadros y figuras y mediante el color resaltar las cantidades correctas, juegos matemáticos.

En tres de los cuadernos que revisamos los niños antes de iniciar el trabajo anotaban el objetivo.

Por otra parte, en las fichas que los maestros elaboraron aparece el nivel, el área, el tema, los contenidos, el nombre de la escuela, las instrucciones, el tipo de ficha y en algunas, el objetivo. Muy parecido a lo que viene indicado en las fichas de PROMECE. Esto quiere decir que el maestro no desconoce del todo algunos de los lineamientos que se deben seguir al elaborar una ficha didáctica; pero en pocas ocasiones las pone en práctica.

Si consideramos las cifras de la tabla № 6, nos damos cuenta de que la elaboración de fichas es escasa, pero estos educadores sí llevan a los niños a escribir, por ello criticaron el abuso en las fotocopias y de las fichas, y el deterioro que se está presentando de la expresión escrita y lectura.
Según los cuadernos revisados y lo que opinaron los niños, ¿qué es lo que más utilizan los maestros para trabajar las diferentes lecciones? En la tabla № 15 se pueden observar las diferentes actividades que se llevan a cabo.

Tabla № 15

Tipo de actividades que más llevan a cabo los educadores con los niños, según la revisión de cuadernos

Actividades

C.E.E.

Buscar palabras en el diccionario, para estudiar vocabulario desconocido.

Confeccionar resúmenes con la ayuda de una guía de preguntas o resumiendo directamente del libro de texto o de un material fotocopiado dado por el educador.

Dibujos de los niños y el colorear los mismos.

Responder cuestionarios con diferentes tipos de preguntas.

Resolver ejercicios de: completar frases y oraciones, parear, asociar, identificar figuras, dar su nombre y significado, entre otros.

Hacer comparaciones y completar cuadros.

Completar dibujos y escribir el nombre de sus partes.

Escribir al lado de imágenes su significado o interpretar lo que significan.

Resolver estudios dirigidos.

Resolver crucigramas.

Desarrollar prácticas para resolver problemas y hacer cálculos.

Hacer lecturas para reflexión y comentarios.

Hacer lecturas con ejercicios de comprensión.

Trabajos en grupos con la ayuda de una ficha o resumen dado por el educador.

Construir ayudado con recortes de periódico.

Resolver palabragramas.

Comentar frases.

Seleccionar respuestas correctas.

Construir oraciones.

Observar secuencias e interpretar por escrito su contenido.

Llevar a cabo trabajos en subgrupos.

Elaborar mapas conceptuales.

Colorear mapas.

Investigar y responder en el cuaderno.

Llevar a cabo experimentos.

Crear historias y cuentos.

Leer poemas y versos para reflexionar y escri-

bir en torno a ello.

2

Llevar a cabo dramatizaciones.

1


Nota: Es importante dejar claro que los educadores además de estas actividades desarrollaron otras que fueron citadas por los niños, cuando los entrevistamos y que los educadores indicaron también, entre ellas: juegos, dinámicas, elaboración de maquetas, copiar de la pizarra, desarrollar exposiciones después de haber trabajado en subgrupos, copiar lo que dicta el educador, etc.

\section{Conclusiones}

Los educadores coinciden al afirmar que las fichas son un recurso valioso en los procesos de enseñanza aprendizaje, puesto que permiten fortalecer una serie de principios en los niños y el desarrollo de contenidos de una manera clara y creativa; pero también son concientes de que una ficha como técnica didáctica requiere de ciertos requisitos para que sea reconocida como tal; entre ellos, uno de los más importantes es el que la ficha sea planificada en función del alumno, dado que la intención original de estas es, en la medida de lo posible, individualizar la enseñanza. Concuerdan en que no puede ser un documento improvisado, pues requiere ser concisa, sin ambigüedad y atractiva para los niños.

Los educadores con los que desarrollamos la investigación consideran que existe abuso al utilizar material (fotocopias) que no cuenta con los requisitos para ser ubicado como ficha didáctica, de ahí que no solo les preocupa la reacción de los padres ante eso, sino que también la respuesta de los niños especialmente cuando reciben Estudios Sociales y Ciencias.

Los maestros encuentran que muchas veces se utiliza material fotocopiado por comodidad, abundancia de fotocopiadoras, falta de tiempo y porque confeccionar las fichas didácticas conlleva mucho trabajo.

\section{Notas}

1. Este es un material que no cuenta con las características que debe tener una ficha didáctica.

2. Este es un material que no cuenta con las características que debe tener una ficha didáctica.

\section{Referencias bibliográficas}

Campos, Ana Victoria "Entrevista directa”. (San Ramón, Alajuela, 10 de octubre, 2001).

Cárdenas, Elizabeth "Entrevista directa". (San Ramón, Alajuela, 29 de agosto, 2001).

Colegio de Licenciados y Profesores. Fichas de Trabajo I-II. Ciclo Educación General Básica. San José, Costa Rica. 1988.

Colegio Patriarca San José. Enseñanza Personalizada. Costa Rica: documento mimeografiado. 1979.

Dottrens, Robert. La enseñanza individualizada. Buenos Aires, Argentina: Editorial Kapelusz. 1959.

Dottrens, Robert. La enseñanza personalizada. Buenos Aires, Argentina: Editorial Kapelusz. 1973.

Fernini, María Rita. Hacia una educación personalizada. 11 $1^{\mathrm{a}}$ edición. México: Edicol. 1979.

Herrera, Julia "Entrevista directa". (San Ramón, Alajuela, 22 de agosto, 2001). 
Jiménez, Anselmo "Entrevista directa". (San Ramón, Alajuela, 6 de agosto, 2001).

Ministerio de Educación Pública. Enseñanza personalizada. San José, Costa Rica: Departamento de publicaciones. 1983.

Palma, Rosa "Entrevista directa". (San Ramón, Alajuela, 22 de agosto, 2001).
Rosa, Gino Di ¿Cómo usamos las fichas? Buenos Aires, Argentina: Editorial Kapelusz. 1974.

Valerio, Cecilia "Entrevista directa". (San Ramón, Alajuela, 8 de agosto, 2001).

Valero, José María. Educación personalizada. México: Editorial Progreso. 1975.

Gilberto Chavarría Chavarría Docente de la Sede de Occidente Universidad de Costa Rica

Alicia Alfaro Valverde Es miembro del Consejo Científico de la Sede de Occidente de la Universidad de Costa Rica 